\title{
Thermodynamic and kinetic description of the second order phase transitions
}

\author{
Yu.L.Klimontovich* \\ Department of Physics, M.V.Lomonosov Moscow State University, \\ Vorob'evy gory, 119899 Moscow, Russia
}

Received November 19, 1999

Thermodynamic and kinetic description of phase transitions for the model of ferroelectrics based on the kinetic equation for the distribution function of values of the "order parameter", coordinates and time is considered.

For one-domain ferroelectrics, the self-consistent approximation for the first moment is used. The kinetic equation is reduced to the relaxation GinsburgLandau equation. The susceptibility is governed by the Curie law and the heat capacity has the jump.

Calculations are carried out for one-domain and polydomain ferroelectrics. In the first case, the self-consistent approximation for the first moment is used. In the second case, the self-consistent approximation for the second moment is carried out. In the last case, there is the jump of the susceptibility. The heat capacity is governed by the Curie law.

It is also shown that the Ornstein-Zernike formula is valid not for the space correlator of fluctuations but only for the temporal spectral density of the space correlator at zero frequency.

In the kinetic theory of the phase transition, all physical characteristics at the critical point have got finite values. Thus, the problem of the "infinities" is absent.

Key words: phase transitions, self-consistent approximations, problem of "infinities", coherent fluctuations, thermodynamic functions, critical point

PACS: $64.60 .-i, 64.60 . F r, 64.60 . \mathrm{My}, 65.50 . t m$

\section{Introduction}

After Gibbs, Van der Waals, Ornstein-Zermike and Weiss in the Landau paper [1], (see also in [2]) the general phenomenological theory of phase transitions was developed. It also serves as the basis for the modern fluctuation theory of phase transitions [2-7]. In this theory, the behaviour of thermodynamic functions in the critical region is characterized by eight critical indexes which are connected by means of five general relations. The sixth relation based on "the scale invariance hypothesis"

*E-mail: ylklim@hklim.phys.msu.su 
was formulated by Kadanoff, Patashinskii-Pokrovskii. In Wilson theory, the special perturbation theory method was developed allowing one to calculate the critical indexes in a better agreement with experimental data. However, the susceptibility and the correlation radius at the critical point, as well as in the Landau theory, are infinite. Only the "rate" of their increasing to infinity varies. Thus, the question about the thermodynamical description of a critical point remains open.

In recent years, in the theory of phase transitions in polymers and spin glasses, many new questions arose, to which only qualitative answers can be given now (see in [8] and [9]). In this situation, the development of non-traditional methods for the description of the phase transitions is natural.

In the present paper, the description of the second order phase transitions is carried out based on the kinetic equation for the distribution function of values of internal parameter (in non-symmetrical phase it plays the role of "the order parameter"), coordinates, and time.

Depending on the structure of the system under consideration (monodomain or polydomain) two possible cases of reducing the kinetic equation to the equations for the moments of distribution function are investigated. In the first case, the self-consistent approximation for the first moment is used. Thus, the kinetic equation is reduced to the relaxation Ginsburg-Landau equation, but the latter one possesses two independent dissipative terms. This equation serves as an example of the reaction-diffusion Fisher-Kolmogorov-Petrovsky-Piscunov (FKPP) equation for the first moment of the distribution function. In this case, the variation of the susceptibility in the critical region is governed by the Curie law, and there is a jump of the heat capacity.

For polydomain systems, the first moment is negligible while the second moment approximation is more effective. In this case, there is also the equation of FKPP type, but it has a different structure. For such systems, there is a jump for the susceptibility and for characteristics which are connected with it, namely, for the correlation radius and for the correlation time. The thermal capacity has no jump. In the critical region, it is governed by the Curie law, and it is finite at the critical point.

Based on the kinetic equation, the calculation of the fluctuations of moments of the distribution function is also carried out. The results differ greatly from those received based on the Landau theory. In particular, it is shown that the one-time space correlations of the Ornstein-Zernike type do not exist. The formula similar to the Ornstein-Zernike one takes place only for the temporal spectral density of the space correlator at zero frequency.

The kinetic derivation of the Ornstein-Zernike formula does not seem to be necessary. Really, it can be obtained, but for certain assumptions (!) regarding the structure of the effective Hamilton function, based on the appropriate "canonical Gibbs distribution". The kinetic description allows, however, to remove these restrictions and to receive additional information about temporal evolution of the fluctuations in the critical region.

In the kinetic theory of the second order phase transitions, the "problem of 
infinities" of the thermodynamic functions at the critical point does not exist. There is the opinion that in the vicinity of the critical point the thermodynamic description is violated. This view of the critical state is based, to a great extent, on the LeeYang theorem, according to which, in the thermodynamic limit, the functions such as susceptibility and correlation radius, take infinite values at the critical point.

The Lee-Yang theorem is, of course, very important, as it shows that critical points are singular. However, in real systems having finite volumes, all the thermodynamic functions are finite. For example, it is obvious, that the correlation radius cannot be larger than the volume of the system. The occurrence of infinity in the theory of phase transitions is the consequence of a certain choice of the succession of two limiting transitions: the transition to the critical point $T \rightarrow T_{\mathrm{c}}$ and the thermodynamic limit $(N \rightarrow \infty$ and $V \rightarrow \infty$, but $N / V$ finite). Usually the first one is the thermodynamic limit. The change of the order of limiting transitions, as it was pointed in the book [10], gives an opportunity to avoid the difficulty mentioned above.

It will be shown, that the finiteness of thermodynamic functions at the critical point is one of the consequences of the kinetic theory of phase transitions with allowance for structures "of continuous medium". Relaxation times also take finite values at the critical point.

Firstly, we compare the kinetic description of phase transitions in the Landau theory as well as based on the statistical theory of open systems [11]. In order to represent the results in the clearest form, we use the elementary model of the ferroelectric phase transition (see $[12,13])$.

\section{The relaxation Ginsburg-Landau equation (RGLE)}

Let $X(R, t)$ be the local value of a relative displacement of crystal lattices in ferroelectrics. In the Landau theory for the nonsymmetrical phase it will play the role of the local order parameter.

According to Landau theory (see in [14]) we introduce (by definition!) the effective Hamilton function. For the ferroelectrics model we can represent it in the form:

$$
H_{\text {eff }}=n m \omega_{0}^{2} \int\left\{\frac{1}{2} X^{2}\left[\left(1-a_{\mathrm{f}}\right)+\frac{1}{2} b X^{2}\right]+\frac{g}{2}\left(\frac{\partial X}{\partial R}\right)^{2}\right\} \mathrm{d} R .
$$

Here $n$ is the mean density of the particles. Inclusion of this factor allows us to introduce the effective Hamilton function on one particle. $m$ is the mass of atom, $m \omega_{0}^{2}$ is the hardness, and $\omega_{0}$ is the proper frequency of the vibration of atoms.

The parameter $a_{\mathrm{f}}(T)$ defines the effect of the Lorentz effective field on the hardness. This parameter depends on the temperature. According to Landau theory in the region of the critical point, this dependence is defined by the expression

$$
1-a_{\mathrm{f}}=\frac{T-T_{\mathrm{c}}}{T_{\mathrm{c}}} \equiv \tau
$$


Here $T_{\mathrm{c}}$ is the critical temperature. Thus, the hardness is zero at the critical point. Below the critical point in the nonsymmetrical phase it is negative. The Lorentz field is determined by the small-scale correlations, which are taken into account here as the phenomenological parameter $a_{\mathrm{f}}$.

To get a qualitative notion regarding the behaviour of the system for all temperatures one may represent the function $a_{\mathrm{f}}(T)$ as follows:

$$
1-a_{\mathrm{f}}=\tanh \frac{T-T_{\mathrm{c}}}{\Delta T}
$$

Here $\Delta T$ is the "temperature width" of the transition region.

The parameter $g$ in the expression $H_{\text {eff }}$ characterizes the degree of nonlocality, and, therefore, is nondissipative one.

In order to simplify the comparison with formulas of the Landau-Lifshits course [1976], we write the correspondence rules:

$$
\begin{aligned}
\alpha\left(T-T_{\mathrm{c}}\right) \eta^{2} & \Leftrightarrow n m \omega_{0}^{2} \frac{X^{2}}{2} \frac{T-T_{\mathrm{c}}}{T_{\mathrm{c}}}, \quad b \eta^{4} \Leftrightarrow n m \omega_{0}^{2} b \frac{X^{4}}{4}, \\
g \eta^{2} & \Leftrightarrow n m \omega_{0}^{2} \frac{g}{2} X^{2}, \quad \eta h \Leftrightarrow n m \omega_{0}^{2} X h .
\end{aligned}
$$

The kinetic equation for the local order parameter in the Landau theory (see in [14]) is the result of the functional differentiation of the effective Hamilton function:

$$
\frac{\partial X(R, t)}{\partial t}=-\frac{\gamma}{n m \omega_{0}^{2}} \frac{\delta H_{\mathrm{eff}}}{\delta X(R, t)} .
$$

Here $\gamma$ is the arbitrarily introduced dissipative coefficient. As a result, we obtain the relaxation Ginsburg-Landau equation (RGLE)

$$
\frac{\partial X(R, t)}{\partial t}=-\gamma\left[\frac{T-T_{\mathrm{c}}}{T_{\mathrm{c}}}+b X^{2}(R, t)\right] X(R, t)+\gamma g \frac{\partial^{2} X(R, t)}{\partial R^{2}} .
$$

Note, that the assumption (5) can be justified only in some special cases. It is impossible, in general, to find the kinetic equation by the functional differentiation of the effective Hamilton function $H_{\text {eff }}$.

Note also, that the initial expression (1) for the effective Hamilton function corresponds to the model of continuous medium. In this case, the introduction of the gradient of macroscopic variables into the $H_{\text {eff }}$ and the use of the corresponding "canonic Gibbs distribution" contradicts the theory of irreversible processes.

The RGLE has the form of reaction-diffusion equation but the diffusion coefficient is defined by the expression $D=\gamma g(! ?)$.

\section{Landau and Boltzmann distribution functions}

Consider the case when the space diffusion process in RGLE is the fastest. Then, for the homogeneous state, the effective Hamilton function has the form:

$$
H_{\mathrm{eff}} \equiv N h_{\mathrm{eff}}, \quad h_{\mathrm{eff}}=\frac{m \omega_{0}^{2}}{2} X^{2}\left[\left(1-a_{\mathrm{f}}\right)+\frac{1}{2} b X^{2}\right]
$$


Here we introduced the effective Hamilton function $h_{\text {eff }}$ for one particle.

The expression (7) for the system of bistable elements defines the Landau effective Hamilton function in one-mode approximation. Through it, the "Landau distribution function" is defined by the following expression:

$$
f_{\mathrm{L}}\left(X, a_{\mathrm{f}}, T\right)=\exp \frac{F\left(a_{\mathrm{f}}, T\right)-H_{\mathrm{eff}}\left(X, a_{\mathrm{f}}\right)}{k_{\mathrm{B}} T} .
$$

Consider the free energy per one particle $F\left(a_{\mathrm{f}}, T\right)=N \psi\left(a_{\mathrm{f}}, T\right)$. Then the Landau distribution function $f_{\mathrm{L}}\left(X, a_{\mathrm{f}}, T\right)$ may be represented in the following form:

$$
f_{\mathrm{L}}\left(x, a_{\mathrm{f}}, T\right)=\exp \frac{N\left(\psi\left(a_{\mathrm{f}}, T\right)-h_{\mathrm{eff}}\left(x, a_{\mathrm{f}}\right)\right)}{k_{\mathrm{B}} T} .
$$

Compare it with the appropriate Boltzmann distribution:

$$
f_{\mathrm{B}}\left(x, a_{\mathrm{f}}, T\right)=\exp \frac{\psi\left(a_{\mathrm{f}}, T\right)-h_{\mathrm{eff}}\left(x, a_{\mathrm{f}},\right)}{k_{\mathrm{B}} T} .
$$

It differs from the Landau distribution function by factor $N$ being absent in the exponent. For this reason, for the Boltzmann distribution, the fluctuations are finite for the thermodynamic limit as well.

For the Landau distribution, for the thermodynamic limit, the fluctuations are zero. In this sense the Landau distribution is coherent (or self-consistent). Thus, in the Landau theory, the "coherence" is already introduced in the initial definition of the effective Hamilton function (1).

Via the parameter $a_{\mathrm{f}}$, the Hamilton function $H_{\text {eff }}$ depends on temperatures and, as a consequence, the additional thermodynamic "thermal force" appears [15]:

$$
F_{\mathrm{th}}(T)=-\int \frac{\partial H_{\mathrm{eff}}(X, T)}{\partial T} f\left(X, a_{\mathrm{f}}, T\right) \mathrm{d} X
$$

This leads to the change of the thermodynamic relations. In particular, the famous Helmholtz relation now has the following form:

$$
\frac{\partial F(T)}{\partial T}=-S_{\mathrm{eff}}(T)-F_{\mathrm{th}}(T)
$$

Here $F(T)$ is the Gibbs free energy, the effective entropy $S_{\text {eff }}(T)$ being defined by the Boltzmann distribution $f_{\mathrm{B}}\left(X, a_{\mathrm{f}}, T\right)$. However, there are other new questions arising in the phase transitions theory. Let us illustrate them using as an example the temperature dependence of the heat capacity.

\section{Thermodynamic functions}

In the Landau theory thermodynamic functions are defined through the most probable values $X_{\text {m.p. }}$ of the distribution function (9):

$$
X_{\text {m.p. }}=0, \quad \tau \geqslant 0 ; \quad X_{\text {m.p. }}= \pm \sqrt{|\tau| b}, \quad \tau \leqslant 0 .
$$


At the critical point the jump of the heat capacity takes place. This jump may be expressed through the dimensionless parameter of nonlinearity:

$$
\Delta C=\frac{1}{2} k_{\mathrm{B}} N\left(\frac{1}{X_{T_{\mathrm{c}}}^{2} b}-1\right)>\frac{1}{2} k_{\mathrm{B}} N, \quad X_{T_{\mathrm{c}}}^{2}=\frac{k_{\mathrm{B}} T}{m \omega_{0}^{2}} .
$$

One can see, that in the Landau theory the peak of the function $C(T)$ is absent. In the approximation considered the heat capacity is defined not by the BoltzmannGibbs entropy but by the thermal force. Thus, the Helmholtz relation (12) is replaced by the following expression:

$$
\frac{\partial F\left(X_{\text {m.p. }}, T\right)}{\partial T}=-F_{\text {th }}\left(X_{\text {m.p. }}, T\right), \quad S_{\text {eff }}\left(X_{\text {m.p. }}, T\right)=0 .
$$

The Boltzmann-Gibbs entropy is not taken into account at this level of the description. For this reason it is impossible to reveal the peak of the function, which defines the $\lambda$-point. In order to obtain a more general result, the following should be taken into account.

In the Gibbs theory it is assumed by definition that the thermodynamic functions are the mean values (the first moments) of the corresponding dynamical variable functions. As an example, the internal energy is represented as the mean value of the Hamilton function $U=\langle H\rangle$. There are, however, other possibilities of defining the thermodynamic functions. For example, the internal energy can be defined via the most probable value for the Gibbs distribution of energy values $U=H_{\text {m.p. }}$. These two definitions are equivalent only under the following condition:

$$
\lim _{N \rightarrow \infty} \frac{1}{N}\left(\langle H\rangle-H_{\text {m.p. }}\right)=0 .
$$

In the theory of phase transitions for Landau and Gibbs distribution functions this condition is, in general, not satisfied. Really, in the nonsymmetrical phase the average value $\langle X\rangle=0$, but the most probable value differs from zero and is defined by the expression (13). Thus, the definition of the thermodynamic functions for phase transitions is not unique and there is a possibility for choice. We shall make use of it to calculate the temperature dependence of the heat capacity $C(T)$ in the critical region.

In the paper [15] the calculation of the function $C(T)$ was conducted based on the generalized Helmholtz relation (12). In the Gaussian approach the following result was obtained:

$$
\begin{gathered}
C=\frac{1}{2} k_{\mathrm{B}} N \frac{T_{\mathrm{c}}^{2}}{\left(T-T_{\mathrm{c}}\right)^{2}}, \quad T>T_{\mathrm{c}}, \\
C=\frac{1}{2} k_{\mathrm{B}} N \frac{T_{\mathrm{c}}^{2}}{\left(T-T_{\mathrm{c}}\right)^{2}}+\frac{1}{2} k_{\mathrm{B}} N \frac{1}{X_{T_{\mathrm{c}}}^{2} b}, \quad T<T_{\mathrm{c}} .
\end{gathered}
$$

The second term in the right-hand side coincides with the Landau jump of the heat capacity. There is, however, an additional singular term, which describes the nonmonotonic behaviour of the heat capacity near the point $\lambda$. The value $C(T=$ $\left.T_{\mathrm{c}}\right) \sim 1 /\left(b X_{T}^{2}\right)$ is finite and has the same order as the Landau jump. 
Such a calculation of the function $C(T)$ corresponds to the Gibbs definition of thermodynamic functions, since all the terms in the right-hand part of the formula (12) are the mean values (the first moments) of the Boltzmann distribution (10). However, values $\langle X\rangle, X_{\text {m.p. }}$ in nonsymmetrical phase are essentially different.

It is possible to use "another" method for calculation, when the entropy is defined by Boltzmann distribution, while the thermal force is defined by Landau distribution. For this case we single out the value of the effective Hamilton function for $X=X_{\mathrm{m}}$.p. in the Boltzmann distribution:

$$
h_{\text {eff }}(X)=h_{\text {eff }}\left(X_{\text {m.p. }}\right)+h_{\text {eff }}^{\prime}\left(X-X_{\text {m.p. }}\right) ; \quad h_{\text {eff }}^{\prime}(0)=0 .
$$

Since $h_{\text {eff }}\left(X_{\text {m.p. }}\right)$ is the thermodynamic characteristic, the thermal force in the formula (12) can be replaced by the derivative of the function $h_{\text {eff }}\left(X_{\text {m.p. }}\right)$ with respect to temperature. As a result, the generalized Helmholtz relation will be presented in the following form:

$$
\frac{\partial F(T)}{\partial T}=-S_{\text {eff }}(T)-F_{\text {th }}\left(X_{\text {m.p. }}\right) ; \quad F_{\text {th }}\left(X_{\text {m.p. }}\right)=-N \frac{\partial h_{\text {eff }}\left(X_{\text {m.p. }}\right)}{\partial T} .
$$

The entropy is defined by the Boltzmann distribution with the Hamilton function $h_{\text {eff }}^{\prime}\left(X-X_{\text {m.p. }}\right) ; h_{\text {eff }}^{\prime}(0)=0$. The heat capacity is now determined by the expression:

$$
C(T)=T\left|\frac{\mathrm{d} S_{\text {eff }}(T)}{\mathrm{d} T}\right|+T \frac{\partial F_{\mathrm{th}}\left(x_{\mathrm{m} . \mathrm{p} .}\right)}{\partial T} .
$$

There is the module of the derivative because the heat capacity is positive, but in the region of the $\lambda$-point the entropy increases with the decrease of temperature. As a result, instead of equations (17) and (18) we have the following expressions:

$$
\begin{gathered}
C=\frac{1}{2} k_{\mathrm{B}} N \frac{T_{\mathrm{c}}}{T-T_{\mathrm{c}}}, \quad T>T_{\mathrm{c}}, \\
C=\frac{1}{2} k_{\mathrm{B}} N \frac{T_{\mathrm{c}}}{T_{\mathrm{c}}-T} \quad+\frac{1}{2} k_{\mathrm{B}} N \frac{1}{X_{T_{\mathrm{c}}}^{2} b}, \quad T<T_{\mathrm{c}} .
\end{gathered}
$$

Thus, the singular part of the heat capacity is defined by the Curie law.

\section{Derivation of the Ornstein-Zernike formula based on the Ginsburg-Landau equation}

Let's return to the relaxation Ginsburg-Landau equation (RGLE) (6), which was obtained based on the expression (1) for the effective Hamilton function by the "recipe" (5). Equation (6) is the example of the reaction-diffusion equation, which serves as the basic equation for the description of a different kind of spatio-temporal dissipative structures and, therefore, plays the role of basic equations in the theory of nonequilibrium phase transitions. Equilibrium phase transitions differ by the fact that the temperature plays the role of control parameter for them. 
The RGLE (6) being an example of the reaction-diffusion equation can be obtained independently of the recipe of its derivation which was represented above. It can serve for the description of equilibrium phase transitions. For this, however, instead of equation (6) with only one dissipative coefficient $\gamma$, it is necessary to use a more general equation (see the next section).

We shall calculate the spatio-temporal fluctuations by two different methods which lead to essentially different results and, consequently, to the two different expressions for one-moment correlator of fluctuations. We shall discuss the reason for such double-natured results and give a physical basis for a unique choice of the result.

First, we use the method based on the fluctuation-dissipation relation (FDR) (see paragraph 101 in [14]). According to this method, introduce an additional term in the effective Hamilton function (1) which is defined by the local external force $X(R, t) h(R, t)$ as consequence, in the right-hand part of the (RGLE) (6) an additional term $\gamma h(R, t)$ appears.

If the external force is not present this equation has a partial homogeneous solution (13). Let $\delta X$ be a small deviation from it for temperatures $T>T_{\mathrm{c}}$. Then the corresponding susceptibility is defined by the following expression:

$$
\chi(\omega, k)=\frac{\gamma}{-\mathrm{i} \omega+\gamma \frac{T-T_{\mathrm{c}}}{T_{\mathrm{c}}}+\gamma g k^{2}} .
$$

The spatio-temporal spectral density of fluctuations $\delta X$ in the Landau theory is defined by FDR via the imaginary part of the susceptibility (see paragraph 101 in [14])

$$
(\delta X \delta X)_{\omega, k}=\frac{2}{\omega} \operatorname{Im} \chi(\omega, k) \frac{X_{T}^{2}}{n}, \quad X_{T}^{2}=\frac{\kappa_{\mathrm{B}} T}{m \omega_{0}^{2}} .
$$

From the above formulas it follows that for the symmetrical phase the spectral density of fluctuation is

$$
(\delta X \delta X)_{\omega, k}=\frac{2 \gamma}{\omega^{2}+\left(\gamma \frac{T-T_{\mathrm{c}}}{T_{\mathrm{c}}}+\gamma g k^{2}\right)^{2}} \frac{X_{T}^{2}}{n} .
$$

By carrying out the integration with respect to frequency we find the appropriate spatial spectral density

$$
(\delta X \delta X)_{k}=\frac{1}{1+r_{\mathrm{c}}^{2} k^{2}} \frac{\left\langle(\delta X)^{2}\right\rangle}{n}, \quad\left\langle(\delta X)^{2}\right\rangle=\frac{X_{T}^{2}}{T-T_{\mathrm{c}} / T_{\mathrm{c}}} .
$$

and after the Fourier transformation we obtain the Ornstein-Zernike (OZ) type formula for the space correlation function

$$
\langle\delta X \delta X\rangle_{r}=\frac{1}{n}\left\langle(\delta X)^{2}\right\rangle \frac{1}{4 \pi r_{\mathrm{c}}^{2} r} \exp \left(-\frac{r}{r_{\mathrm{c}}}\right), \quad r=\left|R-R^{\prime}\right| .
$$

The definition for the correlation radius used here is as follows

$$
r_{\mathrm{c}}^{2}=g \chi(\omega=0, k=0)=g \frac{T_{\mathrm{c}}}{T-T_{\mathrm{c}}} .
$$


Recall the Ginsburg-Levanuk parameter, which characterizes the relative role of fluctuations. Since the most probable distance for correlator (28) is $r=r_{\mathrm{c}}$ it is possible to characterize the role of fluctuations by the ratio of the correlator (28) for $r=r_{\mathrm{c}}$ to the square of the order parameter (13). It is defined by the Ginsburg number

$$
G i=\frac{\langle\delta X \delta X\rangle_{r_{\mathrm{c}}}}{X_{\text {m.p. }}^{2}} \sim \frac{X_{T}^{2} b}{n g^{3 / 2}}\left(\frac{T_{\mathrm{c}}}{\left|T-T_{\mathrm{c}}\right|}\right)^{1 / 2} \ll 1 .
$$

From this, the condition of applicability of the Landau theory follows

$$
\frac{\left|T-T_{\mathrm{c}}\right|}{T_{\mathrm{c}}} \gg \frac{\left(X_{T_{\mathrm{c}}}^{2} b\right)^{2}}{n^{2} g^{3}}, \quad T-T_{\mathrm{c}} \ll 0 .
$$

It is based on the formula O-Z (28). However, the O-Z formula was obtained using another way. The derivation which was represented here gives up some unsolved questions.

First, the calculation of fluctuations is carried out using RGLE (6). The latter was obtained by the "recipe" (5). It contains only one dissipative factor $\gamma$. As a result, the spectral line (26) is "nonsymmetric", i.e., the width of the spectral line is defined by the sum of two dissipative factors, the latter being not represented in the structure of the numerator.

Second, it is accepted that FDR (25) is possible to be expressed through the response to either an appropriate external force, or to an appropriate Langevin random source. In some cases both of these possibilities are equivalent. The considered case is, however, not the general one.

Really, the Langevin internal sources reflect the properties of the system under consideration. Their moments in the kinetic theory of fluctuations (see in $[10,11,14]$ ) are determined by the structure of "collision integrals" and don't coincide with that corresponding to the Landau theory. Let us show this.

Designate the Langevin source in RGLE $(6)$ as $y(R, t)$ and express the spectral density (26) via the spectral density of the source $(y y)_{\omega, k}$ :

$$
(\delta X \delta X)_{\omega, k}=\frac{(y y)_{\omega, k}}{\omega^{2}+\left(\gamma \frac{T-T_{\mathrm{c}}}{T_{\mathrm{c}}}+\gamma g k^{2}\right)^{2}} .
$$

We see, that for the coincidence of the formulas (26) and (32), the spectral density of the Langevin source in RGLE (6) must be defined by the following expression:

$$
(y y)_{\omega, k}=2 \gamma \frac{X_{T}^{2}}{n}
$$

Thus, the intensity of noise is defined here only by the dissipative factor $\gamma$ introduced formally in equations (5) and (6). In formula (33) the "diffusion noise", the intensity of which is proportional to the square of the wave vector $k^{2}$, is not taken into account. This makes one doubt about the derivation of $\mathrm{O}-\mathrm{Z}$ formula (28) which was presented above. We shall see that the diffusion noise, if it is taken into account, radically changes the behaviour of fluctuations in the vicinity of the critical point. 
Below the expression for the intensity of the Langevin source in the reaction-diffusion equation (see in $[11,16]$ ), and, as a consequence, in RGLE will be presented.

Correlation radius (29) takes an abnormally large value only in the critical region of temperatures. Far from the critical point either for high, or for low temperatures its value (in any case, based on the Ginsburg-Landau equation) decreases up to molecular scales. Correlations of such a kind cannot ensure the spatio-temporal coherence in nonsymmetrical phase.

In accordance with the width of the spectral line (26), the appropriate relaxation time (see paragraph 101 in [14]) is determined

$$
\tau_{\text {cor }}=\frac{1}{\gamma \frac{T-T_{\mathrm{c}}}{T_{\mathrm{c}}}+\gamma g k^{2}} .
$$

For $k \neq 0$ it stays finite even at the critical point.

The Ginsburg number $G i$ is the correlation parameter for a nonsymmetrical phase. Below we shall introduce the correlation parameter $K$ for all temperatures. Before doing this, however, we shall conduct the alternative calculation of the spatiotemporal correlator based on the kinetic equation.

\section{Calculation of fluctuations based on the kinetic equation}

For kinetic description of the second order phase transitions we shall use, following chapter 18 in [Klimontovich, 1993], and [Klimontovich, 1995], the kinetic equation for the distribution function $f(X, R, t)$ of not only internal variable $X$, but also of coordinates $R$ of bistable elements:

$$
\frac{\partial f(X, R, t)}{\partial t}=\frac{\partial}{\partial X}\left[D_{(x)} \frac{\partial f}{\partial X}+\frac{1}{m \gamma} \frac{\partial h_{\mathrm{eff}}\left(X, a_{\mathrm{f}}\right)}{\partial X} f\right]+D \frac{\partial^{2} f}{\partial R^{2}}
$$

This equation can be obtained using the general method of deriving the kinetic equations (see in $[11,16]$ ). It contains two dissipative terms which are defined by redistribution of bistable elements in space $R$ with the diffusion coefficient $D$, and redistribution in the space $X$ with the diffusion coefficient $D_{(x)}$, respectively. For simplicity it is easier to suppose that the two diffusion coefficients are equal to each other. In general case there is, of course, an additional parameter $D / D_{(x)}$.

If relaxation time $\tau_{\mathrm{D}}=L^{2} / D$ is much shorter than the other relaxation times the equation (35) can be replaced by the one for the distribution function $f(X, t)$ :

$$
\frac{\partial f(X, t)}{\partial t}=\frac{\partial}{\partial X}\left[D_{(x)} \frac{\partial f}{\partial X}+\frac{1}{m \gamma} \frac{\partial h_{\mathrm{eff}}\left(X, a_{\mathrm{f}}\right)}{\partial X} f\right] .
$$

Its equilibrium solution for the homogeneous distribution coincides with the Boltzmann distribution (10). Note, that the Fokker-Planck equation (36) is used in the theory of phase transitions (see chapter 13 in [5], and chapter 21 in [10]). Here, however, the kinetic equation (35), being one of the basic equations of the statistical theory of open systems (chapter 18 in [11], and [16]) serves as the basis of 
the kinetic theory of phase transitions. It can also serve as the basis of the kinetic theory of fluctuations.

As is well-known, calculation of kinetic fluctuations is carried out (see in $[10,11$, 14]), using the two equivalent methods. Practically it is more convenient to use the Langevin method when the random source is introduced into the kinetic equation (35):

$$
\frac{\partial f(X, R, t)}{\partial t}=\frac{\partial}{\partial X}\left[D_{(x)} \frac{\partial f}{\partial X}+\frac{1}{m \gamma} \frac{\partial h_{\mathrm{eff}}\left(X, a_{\mathrm{f}}\right)}{\partial X} f\right]+D \frac{\partial^{2} f}{\partial R^{2}}+y(X, R, t) .
$$

Let's return to the "dynamical" kinetic equation (35). In the first-moment approximation, the distribution function $f(X, R, t)$ may be represented in the following form:

$$
f(X, R, t,)=\delta(X-X(R, t,)), \quad\langle X\rangle=X(R, t) .
$$

In this case, the equation (35) is reduced to the reaction-diffusion equation (FKPP) for the first moment, i.e., to the function $X(R, t)$ :

$$
\frac{\partial X(R, t)}{\partial t}=-\frac{\omega_{0}^{2}}{\gamma}\left[\frac{T-T_{\mathrm{c}}}{T_{\mathrm{c}}}+b X^{2}(R, t)\right] X(R, t)+D \frac{\partial^{2} X(R, t)}{\partial R^{2}} .
$$

It differs from RGLE (6) by the structure of dissipative terms.

With the help of the equation (39) it is possible to describe, for example, the structure of domain walls in ferroelectrics (see in [13]). In the stationary onedimensional state ( $R$ is parallel to $y$ ) the solution is defined by the following expression:

$$
X(y)=-\left\langle\frac{T_{\mathrm{c}}-T}{T_{\mathrm{c}} b}\right\rangle^{\frac{1}{2}} \tanh \frac{y}{d} .
$$

The width of the wall is defined by the formula:

$$
d=X_{T}\left(2 \frac{T_{\mathrm{c}}}{T_{\mathrm{c}}-T}\right)^{\frac{1}{2}}, \quad X_{T}^{2}=\frac{k T}{m \omega_{0}^{2}} .
$$

The ratio of the thickness of the wall $d$ to the "amplitude" for $X(y=-\infty)$ is defined by the following expression:

$$
\frac{d}{X(y=-\infty)}=\left(2 X_{T}^{2} b\right)^{1 / 2} \frac{T_{\mathrm{c}}}{T_{\mathrm{c}}-T}
$$

While approaching a critical point this ratio is increased by the Curie law. In the opposite case $\left(T \ll T_{\mathrm{c}}\right)$ it is proportional to the small dimensionless parameter $\left(X_{T}^{2} b\right)^{1 / 2}$.

At the critical point, the formula (42) loses its sense. To obtain the corresponding result at the critical point we must use the kinetic equation (37) with the Langevin source (see (19.6.4) in [11]), the appropriate moments of which are defined by expressions (see (19.4.14) in [11]), or (9.2) in [16]). 
From this equation (38) for pulsing function in the approximation of the first moment we obtain the equation FKPP (39), which corresponds to RGLE (6), but now with the Langevin source already. Thus, the noise intensity is defined as a sum of "reaction" and diffusion terms.

We solve the obtained Langevin equation in the linear approximation with respect to fluctuations $\delta X(R, t)=\int X \delta f(X, R, t) \mathrm{d} X$. By solving this equation for $T>T_{\mathrm{c}}$, we get the following expression for spatio-temporal spectral density of fluctuations $\delta X(R, t)$ :

$$
(\delta X \delta X)_{\omega, k}=\frac{2\left(\gamma_{(x)}+D k^{2}\right)}{\omega^{2}+\left(\gamma_{(x)}+D k^{2}\right)^{2}} \cdot \frac{1}{n}\left\langle(\delta X)^{2}\right\rangle .
$$

We denote the "reaction" dissipative coefficient as $\gamma_{(x)}$ at $T>T_{\mathrm{c}}$ and dispersion as $\left\langle(\delta X)^{2}\right\rangle$ for the Boltzmann distribution according to Gaussian approach

$$
\gamma_{(x)}=\frac{T-T_{\mathrm{c}}}{T_{\mathrm{c}}} \frac{\omega_{0}^{2}}{\gamma}, \quad\left\langle(\delta X)^{2}\right\rangle=\frac{k_{\mathrm{B}} T}{m \omega_{0}^{2}} \frac{T_{\mathrm{c}}}{T-T_{\mathrm{c}}} \equiv X_{T}^{2} \frac{T_{\mathrm{c}}}{T-T_{\mathrm{c}}} .
$$

Spectral line (43) is more symmetric than spectral line (26) because both dissipative terms in it are taken into account on equal rights. As a consequence, the spatial density of fluctuations and the spatial correlation functions are defined by other formulas:

$$
(\delta X \delta X)_{k}=\frac{1}{n}\left\langle(\delta X)^{2}\right\rangle ; \quad\langle\delta X \delta X\rangle_{R, R^{\prime}}=\frac{1}{n}\left\langle(\delta X)^{2}\right\rangle \delta\left(R-R^{\prime}\right) .
$$

Thus, the spatial correlation function is not defined now by the O-Z formula. It differs from zero only in the volume of a point of continuous medium (in physically infinitesimal volume $\left.V_{\mathrm{ph}}\right)$, as the function $\left.\delta\left(R-R^{\prime}\right)\right|_{R=R^{\prime}}=V_{\mathrm{ph}}^{-1}$. As a result, for one-point correlator $\left\langle(\delta x)^{2}\right\rangle$ we have the following expression:

$$
\langle\delta X \delta X\rangle_{R=R^{\prime}}=\frac{1}{N_{\mathrm{ph}}}\left\langle(\delta X)^{2}\right\rangle .
$$

Here $N_{\mathrm{ph}}=n V_{\mathrm{ph}}$ is a number of particles in a physically infinitesimal volume. Formula (46) shows, that the dispersion of fluctuations which are smoothed over the volume of a "point" of "continuous medium" is $N_{\mathrm{ph}}$ times less than for the Boltzmann distribution. Thus, the spatial correlator now has the form (45).

The O-Z formula, however, is important for the kinetic theory as well. Now it is connected not with the integral for the spectral line at the frequency $\omega$, but with the spectral line for zero frequency. Let's show this.

From equation (43) the equality follows:

$$
(\delta X \delta X)_{\omega=0, k} \gamma_{(x)}=\frac{2}{1+r_{\mathrm{c}}^{2} k^{2}} \cdot \frac{1}{n}\left\langle(\delta X)^{2}\right\rangle .
$$

Here, the designation (41), which was introduced earlier for the correlation radius, is used. We shall carry out the integration with respect to the wave number in it. 
As a result, we get the following expression:

$$
(\delta X \delta X)_{\omega=0, r} \gamma_{(x)}=\frac{1}{n}\left\langle(\delta X)^{2}\right\rangle \frac{1}{2 \pi r_{\mathrm{c}}^{2} r} \exp \left(-\frac{r}{r_{\mathrm{c}}}\right) ; \quad r_{\mathrm{c}}^{2}=X_{T}^{2} \frac{T_{\mathrm{c}}}{T-T_{\mathrm{c}}} .
$$

The right-hand part in it coincides with the O-Z formula (28) for the spatial correlator of fluctuations.

Thus, the O-Z formula defines the spatial correlation of the temporal spectral density at zero frequency. For this reason, the meaning of the formula $\mathrm{O}-\mathrm{Z}$ in the theory of phase transitions is changed.

The results obtained are based on the solution of the kinetic equation with a Langevin source. The O-Z formula can be also obtained directly using the "Gibbs distribution" with the function $H_{\text {eff }}$. However, this distribution is not the equilibrium solution of the kinetic equation, but it "was introduced by definition". In a separate paper it will be shown that temporal evolution to the $O-Z$ distribution, as well as the Debye distribution in the plasma theory, is described by the equations of the structure being different from that in the theory of the second order phase transitions.

To conclude this section, we shall compare some results of calculating the fluctuations by two different methods, which were presented above. The comparison of results $(28)$ and (48) allows one to introduce a new correlation parameter $K_{n}^{(\omega=0)}$, which is an analogy of the nonsymmetrical phase of Ginsburg parameter $G i$ :

$$
K_{n}^{(\omega=0)}=\frac{(\delta X \delta X)_{\omega=0, r_{\mathrm{c}}} \gamma_{(x)}}{X_{\mathrm{m} . \mathrm{p} .}^{2}} \sim \frac{X_{T_{\mathrm{c}}}^{2} b}{n r_{\mathrm{c}}^{3}}\left(\frac{T_{\mathrm{c}}}{\left|T-T_{\mathrm{c}}\right|}\right)^{2}
$$

Below, we shall give an example of a unified definition of the correlation parameter for all temperatures.

It is possible to consider the spectral density (43) as the fluctuation-dissipation relation (FDR). In order to compare it with the FDR (25), we first write the expression for a response to a Langevin source. It has the following form:

$$
\chi_{(y)}(\omega, k)=\frac{1}{-\mathrm{i} \omega+\gamma_{(x)}+D k^{2}} .
$$

Instead of the FDR (25) we now have (for $T-T_{\mathrm{c}}>0$ ) the following expression:

$$
(\delta X \delta X)_{\omega, k}=\frac{1}{\omega} \operatorname{Im} \chi(\omega, k)(y y)_{\omega, k} .
$$

Here the designation for the spectral density of the Langevin source is introduced:

$$
(y y)_{\omega, k}=2\left(\gamma_{(x)}+D k^{2}\right) \frac{\left\langle(\delta X)^{2}\right\rangle}{n} .
$$

The introduction of it in equation (51) results in the formula (43) for the spatiotemporal spectral density. Thus, the form of FDR of the model considered here is essentially different from the form of FDR (25) in the Landau theory. 
The results obtained are valid only in the linear approximation over fluctuations and are consequently justified only far from the critical point. To obtain the results for the critical point it is necessary to use a more general solution of the kinetic equation (35) .

Based on the equation (35) we obtain a chain of equations for the moments $\left\langle X^{n}\right\rangle$ and, therefore, the "problem of closure" appears. In the self-consistent approximation, when $\left\langle X^{n}\right\rangle=\langle X\rangle^{n}$, we get a closed equation for the first moment $\langle X\rangle$. The RGLE (39) serves as an example. Now consider other possible methods of closure.

\section{Approximation of the second moment. Polydomain ferroelectrics}

Consider an ensemble of ferroelectrics in which the two opposite directions of the polarization vector in the nonsymmetrical phase are equally probable. Its realization is possible if the observation time is much longer than the polarization vector switching time.

In such an ensemble the first moment $\langle X(R, t)\rangle=0$ and it is more natural to use the self-consistent approximation for the second moments $\left\langle X^{2 m}\right\rangle=\left\langle X^{2}\right\rangle^{n}$. We shall show that it also leads to the reaction-diffusion equation, but now for the function $\left\langle X^{2}\right\rangle \equiv\langle E\rangle \equiv E(R, t)$. To this end, instead of expression (38) we shall use the following distribution function:

$$
f(X, R, t)=\frac{1}{2}(\delta(X-\sqrt{\langle E\rangle})+\delta(X+\sqrt{\langle E\rangle})),
$$

for which all odd moments are equal to zero, and the second moment $\left\langle X^{2}\right\rangle \equiv\langle E\rangle \equiv$ $E(R, t)$. The appropriate distribution function of energy $E$ is defined by the following expression:

$$
f(E, R, t)=\int \delta\left(E-X^{2}\right) f(X, R, t) \mathrm{d} X=\delta(E-E(R, t))
$$

The equation for the function $E(R, t)$ is obtained using the kinetic equation (39). In this case it has the following form:

$$
\frac{\partial E(R, t)}{\partial t}=2\left[D_{(x)}-\Gamma\left(\frac{T-T_{\mathrm{c}}}{T_{\mathrm{c}}}+b E(R, t)\right) E(R, t)\right]+D \frac{\partial^{2} E(R, t)}{\partial R^{2}} .
$$

Now we use the stationary solution for the homogeneous state. Under the condition accepted above $D_{(x)}=D=k_{\mathrm{B}} T / m \gamma$, and $\left\langle X^{2}\right\rangle$ is determined by the following expression:

$$
\left\langle X^{2}\right\rangle+\frac{T-T_{\mathrm{c}}}{T_{\mathrm{c}} b}\left\langle X^{2}\right\rangle=\frac{X_{T}^{2}}{b}, \quad E(R, t) \equiv\left\langle X^{2}\right\rangle
$$

Using the dimensionless parameter

$$
X_{T}^{2} b \ll 1
$$


let's write the solution of the last equation for the three selected states:

$$
\left\langle X^{2}\right\rangle= \begin{cases}X_{T}^{2} \frac{T_{\mathrm{c}}}{T-T_{\mathrm{c}}}, & T>T_{\mathrm{c}} \\ X_{T}^{2}\left(\frac{1}{X_{T}^{2} b}\right)^{1 / 2}, & T=T_{\mathrm{c}} \\ \frac{T_{\mathrm{c}}-T}{T_{\mathrm{c}} b}, & T<T_{\mathrm{c}} .\end{cases}
$$

We see that the second moment plays the role of the order parameter for the nonsymmetrical phase. At the critical point it takes a finite value. Above the critical point the function $\left\langle X^{2}\right\rangle$ coincides with the dispersion for the Boltzmann distribution (10).

Thus, the self-consistent approximation of the second moment again results in the RGLE (39), i.e., in the reaction-diffusion equation. However, now the "reaction" term has another structure. One of the consequences is that at the critical point the order parameter differs from zero. As we shall see below, the character of spatiotemporal correlations is also varied.

Consider now a system which consists of a large number of domains. Suppose that in the volume of measurement the number of domains is great. In this case it is possible to use the self-consistent approximation already in the expression for the hardness of atomic oscillators. The latter means that in the kinetic equation (35) (in the member with elastic force) the following substitution is carried out:

$$
\frac{1}{m \gamma} \frac{\partial h_{\mathrm{eff}}\left(X, a_{\mathrm{f}}\right)}{\partial X}=\Gamma\left(\frac{T-T_{\mathrm{c}}}{T_{\mathrm{c}}}+b X^{2}\right) X \rightarrow \Gamma\left(\frac{T-T_{\mathrm{c}}}{T_{\mathrm{c}}}+b\left\langle X^{2}\right\rangle\right) .
$$

In this approximation the kinetic equation (37) has the following form:

$$
\frac{\partial f}{\partial t}+\frac{\partial}{\partial X}\left[D_{(x)} \frac{\partial f}{\partial X}+\Gamma\left(\frac{T-T_{\mathrm{c}}}{T_{\mathrm{c}}}+b E(R, t)\right) X f\right]+D \frac{\partial^{2} f}{\partial R^{2}} .
$$

Together with equation (55) it consists of the closed system of equations for the function $f(X, R, t)$ and average energy $E(R, t)$.

We shall see that in the course of evolution to the equilibrium state there are "fast" and "slow" processes. The evolution of the average energy is a fast process described by the closed equation (55), while the kinetic equation (60) will be used to describe only slow processes. In the latter equation it is possible to use the solution of equation (56) for the function $E(R, t)$. While describing the "slow" processes by the kinetic equation (60), we can represent it in the following form:

$$
\frac{\partial f}{\partial t}=\frac{\partial}{\partial X}\left[D_{(x)} \frac{\partial f}{\partial X}+\frac{D_{(x)}}{\left\langle X^{2}\right\rangle} X f\right]+D \frac{\partial^{2} f}{\partial R^{2}} .
$$

The equilibrium solution of this equation is represented by the Gaussian distribution

$$
f(X)=(2 \pi\langle E\rangle)^{-1 / 2} \exp \left(-\frac{X^{2}}{2\left\langle X^{2}\right\rangle}\right)
$$

Now we can proceed to the calculation of fluctuations. 


\section{Fast and slow fluctuations under phase transitions}

Now we carry out the calculation of fluctuations, and show that the equations (55) and (61) describe two kinds of fluctuations, namely "fast" and "slow".

Using equation (55) with the corresponding Langevin source $\delta y_{(E)}(R, t)$ receive, in the linear approximation, the equation for fluctuation of energy $\delta E(R, t)$. Write this equation for Fourier components:

$$
\frac{\partial \delta E(\omega, k)}{\partial t}+\frac{1}{\tau_{(E)}} \delta E(\omega, k)=\delta y_{(E)}(\omega, k) .
$$

Here the designation for relaxation time of energy fluctuations and an appropriate width of a spectral line are introduced:

$$
\frac{1}{\tau_{(E)}} \equiv \Delta_{(E)}(k)=2 \Gamma\left(\frac{T-T_{\mathrm{c}}}{T_{\mathrm{c}}}+2 b\left\langle X^{2}\right\rangle\right)+D k^{2} .
$$

The average energy $\left\langle X^{2}\right\rangle \equiv\langle E\rangle$ is defined by the solution of equation (56) for all values of temperature. As an example, for temperatures being lower than the critical one the relaxation time is defined by the following expression:

$$
\tau_{(E)}=\frac{1}{2 \Gamma \frac{T_{\tilde{n}}-T}{T_{\mathrm{c}}}+D k^{2}}, \quad \Gamma=\frac{\omega_{0}^{2}}{\gamma} .
$$

We received the expression being the analogue of equation (34) in Landau theory. However, in the approximation of the second moment we have a general expression (64) for relaxation time, which is valid for all values of temperature.

Consider the expression for the response to a Langevin source $\delta y_{(E)}($ R.t), which follows from equation (55) with an appropriate Langevin source and can be determined through the width of a spectral line of energy fluctuations (64):

$$
\chi_{(E)}(\omega, k)=\frac{1}{-\mathrm{i} \omega+\Delta_{(E)}(k)} .
$$

The response is also determined for all values of temperature in a critical region. In particular, for a static response in an unbounded medium $(k=0)$ we have:

$$
\chi_{(E)}(\omega=0, k=0)=\frac{1}{2 \Gamma\left(\frac{T-T_{\mathrm{c}}}{T_{\mathrm{c}}}+2 b\left\langle X^{2}\right\rangle\right)}, \quad \Gamma=\frac{\omega_{0}^{2}}{\gamma} .
$$

Far from the critical point the response is defined by the Curie law, but at the critical point

$$
\chi_{(E)}(\omega=0, k=0)=\frac{1}{4 \Gamma\left(X_{T}^{2} b\right)^{1 / 2}} .
$$

Together with the fast fluctuations there exist slow ones. During their calculation it is possible to assume that the process determined by "fast" fluctuations is already 
established. It gives the ground to use the stationary solution of equation (55) (equation (56)) for the average value of "energy".

For calculating the fast fluctuations we shall use the kinetic equation (61) with the appropriate Langevin source. From it the equation for the function $X(R, t)$ follows:

$$
\frac{\partial X(R, t)}{\partial t}+\frac{D_{(x)}}{\left\langle X^{2}\right\rangle} X(R, t)=D \frac{\partial^{2} X(R, t)}{\partial R^{2}}+y_{(x)}(R, t) .
$$

Average value $\langle X(R, t)\rangle=0$ and, therefore, $\delta X(R, t)=X(R, t)$. As a result, the equation for the Fourier components of function $X(R, t)$ has the following form:

$$
\left(-\mathrm{i} \omega+\frac{1}{\tau_{(X)}}\right) X(\omega, k)=y_{(X)}(\omega, k) .
$$

Here, as well as in equation (63), the designation for the relaxation time of fluctuations $X(R, t)$ and the appropriate width of a spectral line is introduced:

$$
\frac{1}{\tau_{(X)}} \equiv \Delta_{(X)}=\frac{D_{(X)}}{\left\langle X^{2}\right\rangle}+D k^{2} \equiv \frac{D}{\left\langle X^{2}\right\rangle}\left(1+r_{\mathrm{c}}^{2} k^{2}\right)
$$

We take into account the equality $D_{(X)}=D$ and enter the designation for the square of the correlation radius $r_{\mathrm{c}}^{2}=\left\langle X^{2}\right\rangle$. The intensity of Langevin source in equation (63) is defined by expression (19.6.4) in [10], or (9.2) in [11]:

$$
\left(y_{(X)} y_{(X)}\right)_{\omega, k}=2 \Delta_{(X)} \frac{\left\langle X^{2}\right\rangle}{n} .
$$

The appropriate response to $y(\omega, k)$ is defined by the following expression:

$$
\chi_{(X)}(\omega, k)=\frac{1}{-\mathrm{i} \omega+\frac{D}{\left\langle X^{2}\right\rangle}\left(1+r_{\mathrm{c}}^{2} k^{2}\right)},
$$

and, therefore,

$$
\chi_{(X)}(\omega=0, k=0)=\frac{\left\langle X^{2}\right\rangle}{D} \equiv \frac{1}{\Gamma} \frac{\left\langle X^{2}\right\rangle}{X_{T}^{2}} .
$$

Using the solution of equation (56), all of the obtained characteristics can be determined at the critical point as well. Their behaviour in the symmetrical phase (which is higher than the critical point) is qualitatively the same as in the Landau theory. In the nonsymmetrical phase, however, (which is lower than the critical temperature) it is essentially different. Let's show it.

As in the formula (58), we shall distinguish three characteristic states:

$$
\chi_{(X)}(\omega=0, k=0)= \begin{cases}\frac{1}{\Gamma} \frac{T_{\mathrm{c}}}{T-T_{\mathrm{c}}}, & T>T_{\mathrm{c}}, \\ \frac{1}{\Gamma}\left(\frac{1}{X_{T}^{2} b}\right)^{1 / 2}, & T=T_{\mathrm{c}}, \\ \frac{1}{\Gamma} \frac{T_{\mathrm{c}}-T}{T_{\mathrm{c}}} \frac{1}{X_{T}^{2} b}, & T<T_{\mathrm{c}} .\end{cases}
$$

One can see that the susceptibility increases according to the Curie law only as one approaches the critical point on the side of a symmetrical phase. As one approaches 
the critical point on the side of low temperatures, the susceptibility, on the contrary, decreases.

Thus, it is possible to speak about the existence of the "jump of susceptibility". To reveal it we define the temperature dependence of rigidity not by the Landau formula (2), but by the formula (3). It roughly describes the behaviour of different characteristics of phase transitions for all the values of temperature. Then the susceptibility jump will be defined by the expression:

$$
\left.\chi_{(X)}\right|_{T_{\mathrm{c}}-T \gg \Delta T}-\left.\chi_{(X)}\right|_{T-T_{\mathrm{c}} \gg \Delta T}=\frac{1}{\Gamma}\left(\frac{1}{X_{T_{\mathrm{c}}}^{2} b}-1\right) .
$$

Note, that it has the similar structure as the formula (14) for the jump of the heat capacity in the Landau theory. Similar behaviour of a dielectric susceptibility is observed in some types of ferroelectrics (page 88 in [13]).

Below we determine the temperature dependence of the heat capacity for the case considered here. Before this, however, it is necessary to carry out the appropriate determination of relaxation times, screening radius (thickness of domain walls) and correlation radius.

Return to the formula (71). Consider the case of an unbounded system $(k=0)$. In this case the relaxation time is defined by the expression

$$
\tau_{(X)}=\frac{\left\langle X^{2}\right\rangle}{D} \equiv \frac{1}{\Gamma} \frac{\left\langle X^{2}\right\rangle}{X_{T}^{2}}=\chi_{(X)}(\omega=0, k=0)
$$

for all the values of temperature. Using (75) it is possible to determine the relaxation time for the three characteristic states.

Thus, as one approaches the critical point from the side of high temperature, the relaxation time increases according to the Curie law, it is finite at the critical point, and increases on a measure of downturn of temperature. By analogy with the formula (18) it is possible to define the "jump of a relaxation time":

$$
\left.\tau_{(X)}\right|_{T_{\mathrm{c}}-T \gg \Delta T}-\left.\tau_{(X)}\right|_{T-T_{\mathrm{c}} \gg \Delta T}=\frac{1}{\Gamma}\left(\frac{1}{X_{T_{\mathrm{c}}}^{2} b}-1\right) \gg \frac{1}{\Gamma} .
$$

The latter confirms the assumption stated above concerning the existence of fluctuations with large relaxation time, i.e., "slow" (or coherent) fluctuations. Remind, that in the Landau-Khalatnikov theory the relaxation time in the symmetrical phase decreases according to the Curie law (see [14]).

Finally, the correlation radius (or the width of the domain wall) is defined by the expression

$$
r_{\mathrm{c}}^{2}=\frac{D}{\Gamma} \frac{\left\langle X^{2}\right\rangle}{X_{T}^{2}}=\left\langle X^{2}\right\rangle=D \tau_{(X)} .
$$

In accordance with this it is possible to define (qualitatively) the "jump of square of a correlation radius" as well.

Thus, the square of the correlation radius for all $T<T_{\mathrm{c}}$ is much larger than the square of the displacement by the molecular diffusion, as well as the points of the existence of spatial coherence for temperatures are lower than the critical one. 
Using equation (70) and formula (72) we have the expression for the spatiotemporal spectral density

$$
(X X)_{\omega, k}=\frac{2 \Delta_{(X)}}{\omega^{2}+\left(\Delta_{(X)}\right)^{2}} \frac{\left\langle X^{2}\right\rangle}{n} .
$$

The width of the lines $\Delta_{(X)}$ and the dispersion $\left\langle X^{2}\right\rangle$ are determined by formulas (71) and (56) for all values of temperature. The integration with respect to $\omega$ gives the appropriate expression for the spatial spectral density:

$$
(X X)_{k}=\frac{\left\langle X^{2}\right\rangle}{n} .
$$

Since the right-hand part does not depend on $k$, the spatial correlator and the dispersion are defined by the following expressions:

$$
\langle X X\rangle_{R-R^{\prime}}=\frac{\left\langle X^{2}\right\rangle}{n} \delta\left(R-R^{\prime}\right) ; \quad\langle X X\rangle_{R=R^{\prime}}=\frac{\left\langle X^{2}\right\rangle}{N_{\mathrm{ph}}} .
$$

$\left\langle X^{2}\right\rangle$ is defined by the solution of equation (56) . The coincidence with the corresponding formulas (45) and (46) for "fast" fluctuations takes place only for $T>T_{\mathrm{c}}$.

Here, the Ornstein-Zernike formula is also valid only for the temporal correlator at zero frequency:

$$
(\delta X \delta X)_{\omega=0,\left|R-R^{\prime}\right|} \Delta_{(X)}=\frac{\left\langle X^{2}\right\rangle}{n} \frac{1}{2 \pi r_{\mathrm{c}}^{2}\left|R-R^{\prime}\right|} \exp \left(-\frac{\left|R-R^{\prime}\right|}{r_{\mathrm{c}}}\right) .
$$

The structure of this formula is similar to the one of formula (48). However, the temperature dependence of the correlation radius coincides only for $T>T_{\mathrm{c}}$, while for $T<T_{\text {c }}$ the correlation radius in the formula (48), i.e., for "fast" fluctuations, decreases as the temperature is decreased in accordance with the Curie law. For "slow" fluctuations the correlation radius in accordance with the expression (79) remains a macroscopic characteristic for low temperatures $T \ll T_{\mathrm{c}}$ as well. The latter means that in the nonsymmetrical phase the interaction at temperature $T \ll T_{\text {c }}$ is collective.

The result (83) allows one to introduce the dimensionless correlation parameter for all values of temperature:

$$
K^{(\omega=0)}=\frac{(\delta X \delta X)_{\omega=0, r_{\tilde{n}}} \Delta_{(x)}}{\left\langle X^{2}\right\rangle}=\frac{1}{n r_{\mathrm{c}}^{3}}, \quad r_{\mathrm{c}}^{2}=\left\langle X^{2}\right\rangle .
$$

In the symmetrical (index " $s$ ") phase $\left(T>T_{\mathrm{c}}\right)$ :

$$
K^{(\omega=0)}=K_{s}^{(\omega=0)}=\frac{1}{n r_{\mathrm{c}}^{3}}, \quad r_{\mathrm{c}}^{2}=X_{T}^{2} \frac{T_{\mathrm{c}}}{T-T_{\mathrm{c}}}
$$

and, consequently, coincides with the result for "fast" fluctuations. At the critical point the correlation parameter is defined by the following expression:

$$
K^{(\omega=0)}\left(T=T_{\mathrm{c}}\right)=\frac{1}{n r_{\mathrm{c}}^{3}}, \quad r_{\mathrm{c}}^{2}=X_{T}^{2}\left(\frac{1}{X_{T}^{2} b}\right)^{1 / 2}
$$


Finally, for "slow" fluctuations in a nonsymmetrical phase we receive the following expression:

$$
K^{(\omega=0)}=K_{n}^{(\omega=0)}=\frac{1}{n r_{\mathrm{c}}^{3}}, \quad r_{\mathrm{c}}^{2}=X_{T}^{2} \frac{T_{\tilde{n}}-T}{T_{\mathrm{c}}} \frac{1}{X_{T}^{2} b} .
$$

And as above, for the qualitative representation of evolution of the correlation parameter during the phase transition we define the temperature dependence of rigidity not by the Landau formula (2), but by the formula (3). Then, by analogy with formulas (76) and (78), it is possible to define the jump of the correlation radius in the course of transition from a symmetrical phase to a nonsymmetrical one.

Thus, during the phase transition (in the process of temperature decrease), the correlation parameter decreases monotonously. The latter means the reduction of the role of fluctuations and, consequently, the increase of the degree of coherence during the transition to a nonsymmetrical phase.

The latter shows, that in the process of temperature decreasing, the condition of applicability of the self-consistent approximation is improved. When the transition region is narrow enough, one may speak of the jump of correlation parameters.

Since the maximum value of the distribution function (62) is equal to zero, then the heat capacity during the phase transition is defined by the formula

$$
C(T)=T\left|\frac{\mathrm{d} S_{\text {eff }}(T)}{\mathrm{d} T}\right|, \quad S_{\text {eff }}(T)=\frac{1}{2} k_{\mathrm{B}} N\left(\ln \left(2 \pi\left\langle X^{2}\right\rangle+1\right) .\right.
$$

As a result, we have the following expression for the heat capacity:

$$
C(T)=\frac{1}{2} k_{\mathrm{B}} N T \frac{1}{\left\langle X^{2}\right\rangle}\left|\frac{\mathrm{d}\left\langle X^{2}\right\rangle}{\mathrm{d} T}\right|,
$$

which is valid for all values of temperature. From this expression, in particular, it follows, that as one approaches the critical point the heat capacity increases according to the Curie law:

$$
C(T)=\frac{1}{2} k_{\mathrm{B}} N \frac{T_{\mathrm{c}}}{\left|T-T_{\mathrm{c}}\right|},
$$

and at the critical point

$$
C\left(T=T_{\mathrm{c}}\right)=\frac{1}{2} k_{\mathrm{B}} N \frac{1}{\sqrt{X_{T}^{2} b}} .
$$

One can see, that now the jump of the heat capacity is equal to zero and the function $C(T)$ is the symmetrical function of temperature with a finite value at the critical point.

Thus, for polydomain systems, the jump takes place not for the heat capacity, but for a susceptibility and for characteristics connected with it, for example, for the correlation radius. The similar behaviour in ferroelectrics is described on page 88 in [13]. It is interesting that the similar behaviour of the susceptibility and the heat capacity was observed in the course of the phase transitions in the compound $\mathrm{Ni}_{52.3} \mathrm{Mn}_{23.7} \mathrm{Ga}_{25}$ (the private communication with A.N.Vasil'ev). 


\section{Conclusion}

\subsection{Some final remarks}

Thus, we have seen in the theory of phase transitions based on the kinetic equation (35), the characteristics of the phase transitions are finite for all values of temperature. Therefore, the "problem of infinities" of the susceptibility, the heat capacity, and the correlation radius at the critical point is solved.

In the kinetic theory of phase transitions there are two limit cases. In the first of them, the "order parameter" is connected with the first moment and calculations of either dynamical or statistical characteristics are carried out based on the RGLE or the corresponding reaction-diffusion equations. To calculate the fluctuations it is necessary to determine the corresponding Langevin sources.

In the second case, such as in polydomain systems, when the first moment is equal to zero, the approximate equation for the second moment follows from the kinetic equation. Below the critical point it plays the role of "order parameter". In this case the "nonstandard behaviour" of the main characteristics of phase transitions, i.e., the susceptibility and the characteristics connected with it, exhibit a jump. The heat capacity in this case has no jump being symmetrical and taking a finite value at the critical point.

\subsection{Physically infinitesimal scales}

Space correlators in both cases are $\delta$-correlated. The "width" of $\delta$-function is defined by a physically indefinite scale, determining the size of "points of continuous medium". The characteristic size of a domain $L$ is macroscopic for all temperatures $T<T_{\mathrm{c}}$. The width of wall $d$ is macroscopic only in the critical region.

Everything stated above allows one to use a model of continuous medium for the description of phase transitions. Then, following [10], and [11], the physically infinitesimal scale for the critical region can be defined by the following relations:

$$
l_{\mathrm{ph}} \sim \frac{d}{N^{1 / 5}}, \quad N \sim n d^{3} .
$$

Here the designation for the number of particles in volume $d^{3}$ is introduced.

Remind that simultaneous spatial correlators are proportional to the function $\delta\left(R-R^{\prime}\right)$. For this reason the relative dispersion of kinetic fluctuations is small by virtue of the fact, that in the "point" of continuous medium there are many particles (the equation (46)):

$$
\frac{\langle\delta X \delta X\rangle_{R=R^{\prime}}}{\left\langle(\delta X)^{2}\right\rangle}=\frac{1}{N_{\mathrm{ph}}}=\frac{1}{n l_{\mathrm{ph}}^{3}} \sim \frac{1}{N^{2 / 5}} \ll 1 .
$$

The dispersion $\left\langle(\delta X)^{2}\right\rangle$ is defined by the Boltzmann distribution, and the function $\langle\delta X \delta X\rangle_{R=R^{\prime}}$ is defined through fluctuations of the distribution function.

At $T<T_{\mathrm{c}}$ the size of coherent region, i.e., the size of "natural" domain for polydomain state is defined by the correlation radius of "slow" fluctuations, i.e., $r_{\mathrm{c}} \sim$ 
$\sqrt{1 / \delta}$ for $T<T_{\mathrm{c}}$. Thus, the correlation radius of "slow" fluctuations is macroscopic for all temperatures $T<T_{\mathrm{c}}$. It monotonously increases. For this reason, it ensures the existence of the coherence inside the domains.

It is natural, that collective properties exhibit themselves only in the region of temperatures at which the minimal value of the correlation radius is larger than the physically infinitesimal length $l_{\mathrm{ph}}$. The beginning of the phase transition takes place for the temperature for which the inequality begins to be true:

$$
r_{\mathrm{c}}=\sqrt{\left\langle X^{2}\right\rangle}>l_{\mathrm{ph}}
$$

The quantities $r_{\mathrm{c}},\left\langle X^{2}\right\rangle$ are defined by the formulas (56), (58) and (79). The inequality takes place only for a sufficiently small value of the nonlinearity parameter $b$ (or for a sufficiently small value of the dimensionless parameter $X_{T}^{2} b$ ).

The stated results are valid only for large systems, since there were used the solutions of the kinetic equation received without accounting for the finiteness of the system. For small systems, the additional dependence of characteristics of phase transition on the sizes of all systems appears.

\subsection{Stochastic resonance in the case of the second order phase transition}

It is possible to represent the bistable element of ferroelectrics as a small overdamped particle moving (at $T<T_{c}$ ) in a double-well potential under random Langevin forces resulting from thermal fluctuations. Suppose that this element is exposed to an additive periodic external force. Let this force be smaller than the barrier height.

The stochastic resonance arises when the frequency of the external force is close to the Kramers rate $r_{0} \equiv 1 / \tau_{\text {tr }}$, which defines the mean switching rate in the nonmodulated system [17].

For nonsymmetrical phase of ferroelectrics (for temperatures $T<T_{c}$ ) the Kramers rate is given by the formula

$$
r_{0} \equiv \frac{1}{\tau_{\mathrm{tr}}}=\frac{\Gamma}{2 \pi}\left(\left|U^{\prime \prime}\left(X_{\max }\right)\right| U^{\prime \prime}\left(X_{\min }\right)\right)^{1 / 2} \exp \left(-\frac{\Delta U}{X_{T}^{2}}\right) .
$$

Here $\Delta U=U\left(X_{\max }\right)-U\left(X_{\min }\right)$ is the nonmodulated barrier height.

From the expression for the function $U(X)$ for the nonsymmetrical phase of ferroelectrics it follows that:

$$
\left(\left|U^{\prime \prime}\left(X_{\max }\right)\right| U^{\prime \prime}\left(X_{\min }\right)\right)^{1 / 2}=\sqrt{2} \frac{T_{\mathrm{c}}-T}{T_{\mathrm{c}}} \text { for } T_{\mathrm{c}}>T
$$

and

$$
\Delta U=U\left(X_{\max }\right)-U\left(X_{\min }\right)=\frac{1}{4 b}\left(\frac{T_{\mathrm{c}}-T}{T_{\mathrm{c}}}\right)^{2} .
$$

Taking into account these results, we can represent the expression for the Kramers rate for nonsymmetrical phase of ferroelectrics in the following form:

$$
r_{0} \equiv \frac{1}{\tau_{\mathrm{tr}}}=\frac{\Gamma}{\sqrt{2} \pi} \frac{T_{\mathrm{c}}-T}{T_{\mathrm{c}}} \exp \left(-\frac{1}{4 X_{T}^{2} b}\left(\frac{T_{\mathrm{c}}-T}{T_{\mathrm{c}}}\right)^{2}\right) \text {. }
$$


To obtain the "standard" Kramers rate for the bistable element, it is necessary to make the following substitution in the latter formulas

$$
\frac{T_{\mathrm{c}}-T}{T_{\mathrm{c}}} \rightarrow 1
$$

Now the Kramers rate is defined by the formula

$$
r_{0} \equiv \frac{1}{\tau_{\mathrm{tr}}}=\frac{\Gamma}{\sqrt{2} \pi} \exp \left(-\frac{1}{4 X_{T}^{2} b}\right), \quad X_{T}^{2}=\frac{k T}{m \omega_{0}^{2}} .
$$

We see that in the ferroelectrics in the critical region there is the alternative possibility to control the stochastic resonance. The increase of temperature $\left(T_{\mathrm{c}}-T>0\right)$ decreases the height of the barrier.

In the paper [18] the new phenomenon of the mean switching frequency locking between the states of a bistable system driven by a periodic force was found. Now a new possibility has appeared to observe this effect in the electric circuit with the ferroelectrics elements.

\section{References}

1. Landau L.D. To the theory of phase transitions. // JETP, 1937, vol. 7, p. 19-35.

2. Landau L.D., Lifshits E.M. Statistical Physics. Moscow, Nauka (in Russian).

3. Stanley H.E. Introduction to Phase Transition and Critical Phenomena. Oxford, Clarendon Press, 1971.

4. Patashinskii A.Z., Pokrovskii V.L. Fluctuation Theory of Phase Transitions. Moscow, Nauka, 1982 (in Russian).

5. Ma Sh. Modern Theory of Critical Phenomena. London, Benjamin; Moscow, Mir, 1980.

6. Anisimov M.A. Critical Phenomena in Liquids and Liquid Crystals. Moscow, Nauka, 1987 (in Russian).

7. Balescu R. Equilibrium and Nonequilibrium Statistical Mechanics. New York, John Wiley and Sons, 1975.

8. Grossberg A.Ju., Khokhlov A.R. Statistical Physics of Macromolecules. Moscow, Nauka, 1989 (in Russian).

9. Dotsenko V.S. Physics of spin glass state. // Uspekhi Fiz. Nauk, 1993, vol. 163, p. 137 .

10. Klimontovich Yu.L. Statistical Physics. Moscow, Nauka, 1982; New York, Harwood Academic Publishers, 1986.

11. Klimontovich Yu.L. Statistical Theory of Open Systems. Moscow, Yanus; Dordrecht, Kluwer Academic publishers, 1995.

12. Ginsburg V.L. Theory of ferroelectrics phenomena. // Uspechi Fiz. Nauk, 1949, vol. 38, p. $490-525$.

13. Strukov B.A., Levanuk A.P. Physical fundamentals of ferroelectrics phenomena in crystals. Moscow, Nauka-Fizmatlit, 1995 (in Russian).

14. Lifshits E.M., Pitaevskii L.P. Physical Kinetics. Moscow, Nauka, 1979. 
15. Klimontovich Yu.L. Two alternative descriptions of second order phase transitions: Landau theory and the self-consistent approach. // Physics Letters A, 1996, vol. 210, p. $65-70$.

16. Klimontovich Yu.L. From the Hamiltonian mechanics to a continuous media. Dissipative structures. Criteria of self-organization. // TMP, 1993, vol. 96, p. 385-416.

17. Moss Franck Stochastic Resonance. Saint Louis, Department of Physics, University of Missouri, 1992.

18. Shulgin B., Neiman A., Anishchenko V. Mean switching frequency locking in stochastic bistable systems driven by a periodic force. // Phys. Rev. Lett., 1995, vol. 23, p. 41574160.

\section{Термодинамічний та кінетичний опис фазових переходів другого роду}

\section{Ю.Л.Клімонтович}

Фізичний факультет

Московського державного університету ім. М.В.Ломоносова, Воробйовиє гори, 119899 Москва, Росія

\section{Отримано 19 листопада 1999 р.}

Проведено опис термодинаміки і кінетики фазових переходів моделі сегнетоелектриків на основі кінетичного рівняння для функції розподілу, залежної від “параметра порядку", координат і часу.

Для однодоменних сегнетоелектриків застосовано самоузгоджений підхід до розрахунку першого момента. Кінетичне рівняння зводиться до релаксаційного рівняння Гінзбурга-Ландау. Сприйнятливість описується законом Кюрі, а теплоємність має стрибок.

Розрахунки проведено для однодоменних та полідоменних сегнетоелектриків. У першому випадку використовується самоузгоджене наближення для першого моменту. У другому випадку самоузгоджене наближення робиться для другого моменту. В цьому випадку відзначається стрибок сприйнятливості, а теплоємність описується законом Кюрі.

Показано, що формула Орнштейна-Церніке справедлива не для просторового корелятора флуктуацій, а лише для спектральної густини цієї кореляційної функції при нульовій частоті.

В кінетичній теориї фазових переходів усі фізичні величини мають скінчені значення в критичній точці. Таким чином, немає проблеми "розбіжностей".

Ключові слова: фазові переходи, самоузгоджені наближення, проблема "розбіжностей", когерентні флуктуації, термодинамічні функції, критична точка

PACS: $64.60 .-i, 64.60 . F r, 64.60 . M y, 65.50 . t m$ 